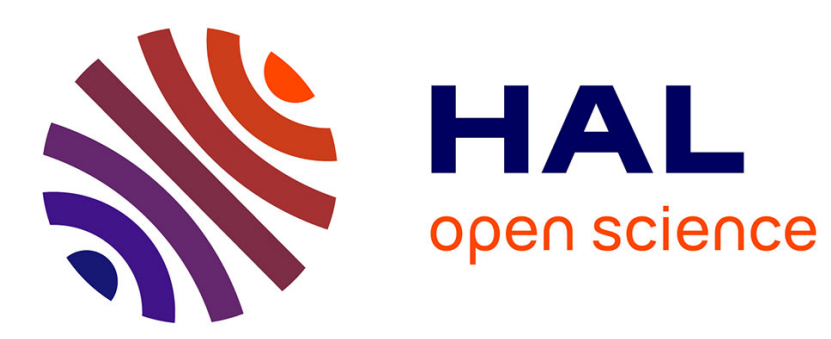

\title{
Exceptional orthogonal polynomials and generalized Schur polynomials
}

Yves Grandati

\section{To cite this version:}

Yves Grandati. Exceptional orthogonal polynomials and generalized Schur polynomials. Journal of Mathematical Physics, 2014, 55 (8), pp.083509. 10.1063/1.4891923 . hal-01513421

\section{HAL Id: hal-01513421 \\ https://hal.univ-lorraine.fr/hal-01513421}

Submitted on 9 May 2017

HAL is a multi-disciplinary open access archive for the deposit and dissemination of scientific research documents, whether they are published or not. The documents may come from teaching and research institutions in France or abroad, or from public or private research centers.
L'archive ouverte pluridisciplinaire HAL, est destinée au dépôt et à la diffusion de documents scientifiques de niveau recherche, publiés ou non, émanant des établissements d'enseignement et de recherche français ou étrangers, des laboratoires publics ou privés. 


\section{Exceptional orthogonal polynomials and generalized Schur polynomials}

Yves Grandati

Citation: Journal of Mathematical Physics 55, 083509 (2014); doi: 10.1063/1.4891923

View online: http://dx.doi.org/10.1063/1.4891923

View Table of Contents: http://aip.scitation.org/toc/jmp/55/8

Published by the American Institute of Physics

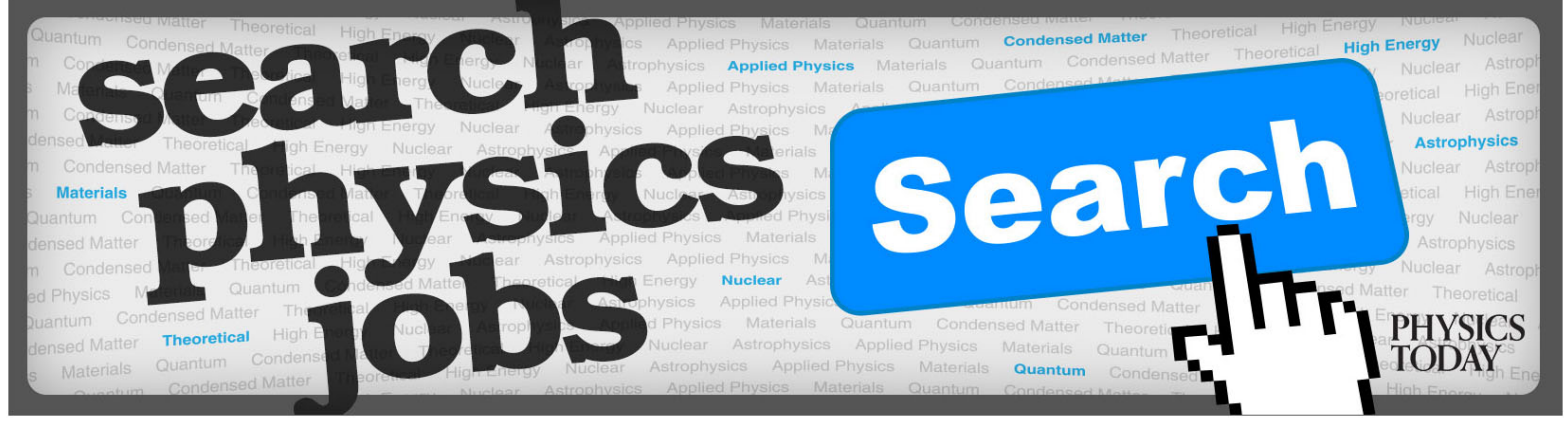




\title{
Exceptional orthogonal polynomials and generalized Schur polynomials
}

\author{
Yves Grandati \\ Equipe BioPhyStat, LCP A2MC, Université de Lorraine, 1 Bd Arago, 57078 Metz, \\ Cedex 3, France
}

(Received 31 January 2014; accepted 21 July 2014; published online 6 August 2014)

\begin{abstract}
We show that the exceptional orthogonal polynomials can be viewed as confluent limits of the generalized Schur polynomials introduced by Sergeev and Veselov. (c) 2014 AIP Publishing LLC. [http://dx.doi.org/10.1063/1.4891923]
\end{abstract}

\section{INTRODUCTION}

The concept of exceptional orthogonal polynomials (EOP) has been introduced five years ago by Gomez-Ullate, Kamran, and Milson. ${ }^{1,2}$ They form families of orthogonal polynomials whose degree sequences present a finite number of gaps but which span a complete basis of their corresponding Hilbert spaces. They appear to be closely related to exactly solvable quantum systems built from translationally shape invariant potentials (TSIP) via chains of Darboux-Bäcklund transformations and this connection has been the subject of an active research during the last years. The exceptional Hermite, Laguerre, and Jacobi polynomials are expressible as Wronskians of the corresponding classical orthogonal polynomials and give, up to a gauge factor, the eigenstates of these extended potentials (see Refs. 3 and 4 and references therein).

In this letter we show that these Wronskians can be viewed as confluent limit of alternants which coincide with the generalized Schur polynomials defined in their seminal paper by Sergeev and Veselov ${ }^{5}$ and recently generalized by Harnad and Lee. ${ }^{6}$ The confluent limit of the generalized Jacobi-Trudy formula established by Sergeev and Veselov is also discussed.

\section{CHAIN OF STATE-DELETING DARBOUX-BÄCKLUND TRANSFORMATIONS (SDDBT)}

\section{A. One step Darboux-Bäcklund transformations}

We consider a one-dimensional Hamiltonian $\widehat{H}=-d^{2} / d x^{2}+V(x), x \in I \subset \mathbb{R}$ and the associated Schrödinger equation

$$
\psi_{E}^{\prime \prime}(x)+(E-V(x)) \psi_{E}(x)=0,
$$

$\psi_{E}(x)$ being a formal eigenfunction of $\widehat{H}$ for the eigenvalue $E$. With Dirichlet boundary conditions on $I, \widehat{H}$ is supposed to admit a discrete spectrum of energies and eigenstates $\left(E_{n}, \psi_{n}\right)_{n \in\left\{0, \ldots, n_{\max }\right\} \subseteq \mathbb{N}}$, where, without loss of generality, we can always take the ground level of $\widehat{H}$ at zero $\left(E_{0}=0\right)$. We suppose also that in the considered domain of eigenvalues, these ones can be indexed by a real spectral parameter $\mu\left(E \rightarrow E_{\mu}, \psi_{E} \rightarrow \psi_{\mu}(x)\right)$, which takes integer values $\mu=n \in \mathbb{N}$ when $E_{n}$ is a bound state energy.

Consider a given solution $\psi_{\nu}(x)$ which is a formal eigenfunction of $\widehat{H}$ for the eigenvalue $E_{\nu}$ without any reference to the boundary conditions (in the following we simply call eigenfunctions such formal eigenfunctions, employing eigenstates for the eigenfunctions satisfying the Dirichlet boundary conditions). We define the first order operator $\widehat{A}\left(w_{v}\right)$ by

$$
\widehat{A}\left(w_{v}\right)=d / d x+w_{v}(x),
$$


where $w_{\mu}(x)=-\psi_{\mu}^{\prime}(x) / \psi_{\mu}(x)$. For $\mu \neq v$, the function defined via the Darboux-Crum formula

$$
\psi_{\mu}^{(v)}=\widehat{A}\left(w_{\nu}\right) \psi_{\lambda}(x)=\frac{W\left(\psi_{\nu}, \psi_{\mu} \mid x\right)}{\psi_{v}(x)},
$$

where $W\left(y_{1}, \ldots, y_{m} \mid x\right)$ denotes the Wronskian of the family of functions $y_{1}, \ldots, y_{m}$,

$$
W\left(y_{1}, \ldots, y_{m} \mid x\right)=\left|\begin{array}{ccc}
y_{1}(x) & \ldots & y_{m}(x) \\
\ldots & & \ldots \\
y_{1}^{(m-1)}(x) & \ldots & y_{m}^{(m-1)}(x)
\end{array}\right|
$$

is then a solution of the Schrödinger equation

$$
\psi_{\mu}^{(v) \prime \prime}(x)+\left(E_{\mu}-V^{(v)}(x)\right) \psi_{\mu}^{(v)}(x)=0,
$$

with the same energy $E_{\mu}$ as in Eq. (1) but with a modified potential

$$
V^{(v)}(x)=V(x)+2 w_{v}^{\prime}(x) .
$$

We call $V^{(v)}(x)$ an extension of $V(x)$ and the correspondence

$$
\left(\begin{array}{c}
V(x) \\
\psi_{\mu}(x)
\end{array}\right) \stackrel{A\left(w_{v}\right)}{\rightarrow}\left(\begin{array}{l}
V^{(v)}(x) \\
\psi_{\mu}^{(v)}(x)
\end{array}\right)
$$

is called a Darboux-Bäcklund Transformations (DBT). The eigenfunction $\psi_{v}$ is the seed function of the DBT $A\left(w_{\nu}\right)$.

Note that $\widehat{A}\left(w_{v}\right)$ annihilates $\psi_{v}$ and consequently the formula Eq. (3) allows to obtain an eigenfunction of $V^{(v)}$ for the eigenvalue $E_{\mu}$ only when $\mu \neq v$. Nevertheless, we can readily verify that $1 / \psi_{\nu}(x)$ is such an eigenfunction. By extension, we then define the "image" by $A\left(w_{v}\right)$ of the seed eigenfunction $\psi_{v}$ itself as

$$
\psi_{v}^{(v)}(x) \sim 1 / \psi_{v}(x)
$$

\section{B. Formal chains of Darboux-Bäcklund transformations}

At the formal level, the DBT can be straightforwardly iterated and a chain of $m$ DBT is simply described by the following scheme:

$$
\left\{\begin{array}{c}
\psi_{\mu} \stackrel{A\left(w_{v_{1}}\right)}{\longmapsto} \psi_{\mu}^{\left(v_{1}\right)} \stackrel{A\left(w_{v_{2}}^{\left(\mathcal{N}_{1}\right)}\right)}{\longmapsto} \psi_{\mu}^{\left(\mathcal{N}_{2}\right)} \ldots \stackrel{A\left(w_{v_{m}}^{\left(\mathcal{N}_{m-1}\right)}\right)}{\longmapsto} \psi_{\mu}^{\left(\mathcal{N}_{m}\right)} \\
V \stackrel{A\left(w_{v_{1}}\right)}{\longmapsto} V^{\left(v_{1}\right)} \stackrel{A\left(w_{v_{2}}^{\left(\mathcal{N}_{1}\right)}\right)}{\longmapsto} V^{\left(\mathcal{N}_{2}\right)} \ldots \stackrel{A\left(w_{v_{m}}^{\left(\mathcal{N}_{m-1}\right)}\right)}{\longmapsto} V^{\left(\mathcal{N}_{m}\right)}
\end{array},\right.
$$

where $\mathcal{N}_{j}$ denotes the $j$-tuple $\left(v_{1}, \ldots, v_{j}\right)$ (with $\mathcal{N}_{1}=v_{1}$ ) of spectral indices which completely characterizes the chain. We note $\left(\mathcal{N}_{m}, v_{m+1}, \ldots, v_{m+k}\right)$ the chain obtained by adding to the chain $\mathcal{N}_{m}$ the DBT associated to the successive eigenfunctions $\psi_{v_{m+1}}^{\left(\mathcal{N}_{m}\right)}, \ldots, \psi_{v_{m+k}}^{\left(\mathcal{N}_{m+k-1}\right)}$. In the following, a capital calligraphic $\mathcal{N}_{m}$ is used for a $m$-tuple of arbitrary real spectral indices, while a capital latin $N_{m}=\left(n_{1}, \ldots, n_{m}\right) \in \mathbb{N}^{m}$ is used for the particular case of a $m$-tuple of integer spectral indices.

$\psi_{\mu}^{\left(\mathcal{N}_{m}\right)}$ is an eigenfunction associated to the eigenvalue $E_{\mu}$ of the potential (see Eq. (6)):

$$
V^{\left(\mathcal{N}_{m}\right)}(x)=V(x)+2 \sum_{j=1}^{m}\left(w_{\nu_{j}}^{\left(\mathcal{N}_{j-1}\right)}(x)\right)^{\prime}=V(x)-2 \sum_{j=1}^{m}\left(\log \left(\psi_{\nu_{j}}^{\left(\mathcal{N}_{j-1}\right)}(x)\right)\right)^{\prime \prime} .
$$

It can be written as (cf. Eq. (3))

$$
\psi_{\mu}^{\left(\mathcal{N}_{m}\right)}(x)=\widehat{A}\left(w_{v_{m}}^{\left(\mathcal{N}_{m-1}\right)}\right) \psi_{\mu}^{\left(\mathcal{N}_{m-1}\right)}(x)=\widehat{A}\left(w_{v_{m}}^{\left(\mathcal{N}_{m-1}\right)}\right) \ldots \widehat{A}\left(w_{\nu_{1}}\right) \psi_{\mu}(x),
$$


that is,

$$
\psi_{\mu}^{\left(\mathcal{N}_{m}\right)}(x)=\frac{W\left(\psi_{\nu_{m}}^{\left(\mathcal{N}_{m-1}\right)}, \psi_{\mu}^{\left(\mathcal{N}_{m-1}\right)} \mid x\right)}{\psi_{v_{m}}^{\left(\mathcal{N}_{m-1}\right)}(x)} .
$$

A chain is non-degenerate if the spectral indices $v_{i}$ of the chain $\mathcal{N}_{m}$ are all distinct and is degenerate if some of them are repeated in the chain. For the non-degenerate chains, Crum has established formulas which give the extended potentials and their eigenfunctions in terms of Wronskians of eigenfunctions of the initial potential. ${ }^{3,7,8}$

\section{Crum's formulas:}

When all the $v_{j}$ and $\mu$ are distinct, we have

$$
\psi_{\mu}^{\left(\mathcal{N}_{m}\right)}(x)=\frac{W^{\left(\mathcal{N}_{m}, \mu\right)}(x)}{W^{\left(\mathcal{N}_{m}\right)}(x)}
$$

and

$$
V^{\left(\mathcal{N}_{m}\right)}(x)=V(x)-2\left(\log W^{\left(\mathcal{N}_{m}\right)}(x)\right)^{\prime \prime},
$$

where $W^{\left(\mathcal{N}_{m}\right)}(x)=W\left(\psi_{v_{1}}, \ldots, \psi_{v_{m}} \mid x\right)$.

The eigenfunctions $\psi_{v_{1}}, \ldots, \psi_{v_{m}}$ of $V$ are called the seed functions of the chain of DBT associated to the $m$-tuple of spectral indices $\mathcal{N}_{m}=\left(v_{1}, \ldots, v_{m}\right)$.

\section{Chains of state-deleting Darboux-Bäcklund transformations and partitions}

In the following, we call state-deleting DBT (SDDBT) every DBT whose seed function is an eigenstate. A chain of SDDBT is then characterized by an $m$-tuple $N_{m}=\left(n_{1}, \ldots, n_{m}\right), n_{i+1}>n_{i}$ $\geq 0$, of distinct positive integers such that the corresponding seed functions $\psi_{n_{i}}$ are eigenstates of the initial potential.

Consider such a chain of SDDBT $N_{m}=\left(n_{1}, \ldots, n_{m}\right)$ with $n_{m}>\ldots>n_{1} \geq 0$. We can equivalently characterize this chain by a partition $\lambda=\left(\lambda_{1}, \ldots, \lambda_{m}\right)$ of length $l(\lambda)=m$, where $\lambda_{1}$ $\geq \ldots \geq \lambda_{m} \geq 0$ and

$$
\lambda_{i}=n_{m-i+1}-m+i
$$

$\lambda$ is a partition of the integer:

$$
|\lambda|=\sum_{i=1}^{m} \lambda_{i}=\sum_{i=1}^{m} n_{i}-m(m-1) / 2 .
$$

Note that, contrarily to the usual convention, we authorize $\lambda$ to contain at its end a string of zeros. The reduced form $\tilde{\lambda}$ of the partition is obtained by suppressing this last string of zeros. If $\lambda$ does not contain such a chain we say that it is an irreducible partition.

To this partition is associated a Young diagram which characterizes the spectral shape of the extension. The $\lambda_{i}$ are the lengths of the columns of the Young diagram starting from the left corner. Following Refs. 4 and 9, we define the double partition $\lambda^{2}$ of $\lambda$ as the partition of length $l\left(\lambda^{2}\right)$ $=2 m$ defined as $\lambda^{2}=\left(\lambda_{1}^{2}, \ldots, \lambda_{m}^{2}\right)$, where we note $\lambda_{i}^{k}$ for $\lambda_{i}$ repeated $k$ times $\left(\lambda_{i}^{k}=\lambda_{i}, \ldots, \lambda_{i}\right)$. We call an Adler partition a partition $\lambda$ whose reduced form, $\widetilde{\lambda}=\left(\lambda_{1}^{m_{1}}, \ldots, \lambda_{k}^{m_{k}}\right)$, is a double partition.

The qualifier state-deleting used above is somewhat abusive, since strictly speaking it has to be reserved to chains leading to regular potentials. Indeed, in this case, the action on the spectrum of the successive DBT based on eigenstates corresponds to suppress at each step the level associated to the used seed function, which then justifies the denomination "state-deleting" DBT.

Krein $^{10}$ and later Adler ${ }^{11}$ have given a necessary and sufficient regularity condition for the final extensions of such non degenerate chains of SDDBT. The Krein-Adler theorem can be rewritten as a structural condition for the partition associated to the chain of $\mathrm{SDDBT}^{4,10,11}$ 
as follows:

\section{Krein-Adler theorem:}

The final extension of the chain of SDDBT associated to the m-tuple $N_{m}=\left(n_{1}, \ldots, n_{m}\right), n_{i+1}>$ $n_{i} \geq 0$, or equivalently to the partition $\lambda=\left(\lambda_{1}^{m_{1}}, \ldots, \lambda_{k}^{m_{k}}, 0^{r}\right),{ }_{i=1}^{k} m_{i}+r=m$, is regular iff $\lambda$ is an Adler partition, that is, iff $m_{i} \in 2 \mathbb{N}, \forall i \in\{1, \ldots, k\}$. The spectrum of the final extension $V^{\left(N_{m}\right)}(x)$ contains only even gaps (gaps constituted by an even number of consecutive missing levels).

Such a chain of SDDBT is said of the Krein-Adler type and in the particular case where $\tilde{\lambda}=0$, the chain is said to be complete.

\section{JACOBI-TRUDI FORMULA FOR EXCEPTIONAL ORTHOGONAL POLYNOMIALS}

\section{A. Primary translationally shape invariant potentials}

Consider a potential $V(x ; a)$ which depends upon a (multi)parameter $a \in \mathbb{R}^{N}$ and which admits a (finite or infinite) bound state spectrum $\left(E_{n}, \psi_{n}\right)_{n \geq 0}$, the ground level being supposed to be zero: $E_{0}(a)=0$. In the framework of supersymmetric quantum mechanics (SUSY QM), such a potential is said to be shape invariant (SIP) ${ }^{12-14}$ if its SUSY partner,

$$
V^{(0)}(x ; a)=V(x ; a)+2 w_{0}^{\prime}(x ; a),
$$

keeps the same functional form as the initial potential. Namely,

$$
V^{(0)}(x ; a)=V(x ; f(a))+R(a),
$$

$R(a) \in \mathbb{R}$ and $f(a) \in \mathbb{R}^{N}$ being two given functions of $a$.

In this case, it can be shown ${ }^{12-14}$ that the complete bound state energy spectrum of $\widehat{H}(a)$ $=-\frac{d^{2}}{d x^{2}}+V(x ; a)$ is given by

$$
E_{n}(a)=\sum_{k=0}^{n-1} R\left(a_{k}\right)=\sum_{i=0}^{n-1} E_{1}\left(a_{i}\right)
$$

where $a_{k}=f^{(k)}(a)=\overbrace{f \circ \ldots \circ f}^{\mathrm{k} \text { times }}(a)$.

As for the corresponding eigenstates, they can be written as

$$
\psi_{n}(x ; a) \propto \widehat{A}^{+}(a) \psi_{n-1}\left(x ; a_{1}\right) \propto \widehat{A}^{+}(a) \ldots \widehat{A}^{+}\left(a_{n-1}\right) \psi_{0}\left(x ; a_{n}\right),
$$

where $\widehat{A}^{+}(a)=-\frac{d}{d x}+w_{0}(x ; a)$.

When $f$ is a simple translation $f(a)=a+\varepsilon, \varepsilon=\left(\varepsilon^{(1)}, \ldots, \varepsilon^{(N)}\right) \in \mathbb{R}^{N}, V$ is said to be translationally shape invariant and we call it a TSIP. For all the known TSIP we have $a \in \mathbb{R}$ (first category TSIP) or $a \in \mathbb{R}^{2}$ (second category TSIP). ${ }^{12,13,15}$

The set of TSIP contains all the potentials classically known to be exactly solvable, i.e., for which we know explicitly the "dispersion relation" ( $E_{n}$ as a function of $\left.n\right)$ and whose the eigenfunctions can be expressed in closed analytical form in terms of elementary transcendental functions: the harmonic, isotonic, Morse, Kepler-Coulomb, Eckart, Darboux-Pöschl-Teller (hyperbolic and trigonometric), and Rosen-Morse (hyperbolic and trigonometric) potentials. These potentials are primary TSIP (PTSIP) from which it is possible in some cases to build infinite towers of secondary TSIP (STSIP) which are extensions of the previous ones and which share the same translational shape invariance properties. $^{16}$

An important feature of the PTSIP is that their eigenfunctions $\psi_{n}$ are equal, up to a gauge factor, to classical orthogonal polynomials in an appropriate variable $z$ (which can be $n$ dependent) and we say that $\psi_{n}$ is quasi-polynomial in this variable. The confining (i.e., diverging at both boundaries of the definition interval) PTSIPs, which then possess an infinite bound state spectrum, are the harmonic, isotonic, and trigonometric Darboux-Pöschl-Teller (TDPT) potentials. For these ones, the gauge factor and the adapted variable are independent of $n$ and their (unnormalized) eigenstates can 
be written as

$$
\psi_{n}(x ; a)=\psi_{0}(x ; a) P_{n}^{a}(z(x)),
$$

where $P_{n}^{a}(z)$ is a monic classical orthogonal polynomial (Hermite, Laguerre, and Jacobi). ${ }^{17,18}$

The $P_{n}^{a}(z)$ satisfy the recursion relation

$$
\left\{\begin{array}{c}
P_{0}^{a}(z)=1 \\
z P_{n}^{a}(z)=P_{n+1}^{a}(z)+p_{n, a} P_{n}^{a}(z)+q_{n, a} P_{n-1}^{a}(z)
\end{array},\right.
$$

with

$$
\begin{gathered}
\left\{\begin{array}{c}
p_{n, a}=0 \\
q_{n, a}=q_{n}=n / 2
\end{array},\right. \text { for the Hermite case, } \\
\left\{\begin{array}{c}
p_{n, a}=p_{n, \alpha}=2 n+1+\alpha \\
q_{n, a}=q_{n, \alpha}=n(n+\alpha)
\end{array},\right. \text { for the Laguerre case, }
\end{gathered}
$$

and

$$
\left\{\begin{array}{c}
p_{n, a}=p_{n, \alpha, \beta}=\frac{\beta^{2}-\alpha^{2}}{(2 n+2+\alpha+\beta)(2 n+\alpha+\beta)} \\
q_{n, a}=q_{n, \alpha, \beta}=\frac{4 n(n+\alpha)(n+\beta)(n+\alpha+\beta)}{(2 n+1+\alpha+\beta)(2 n+\alpha+\beta)^{2}(2 n-1+\alpha+\beta)}
\end{array}\right. \text {, for the Jacobi case. }
$$

In the following, we give a resumed description of the spectral properties of the three confining PTSIP.

\section{Harmonic oscillator} line by

The harmonic oscillator $(\mathrm{HO})$ potential (with zero ground level $\left.E_{0}=0\right)$ ) is defined on the real

$$
V(x ; \omega)=\frac{\omega^{2}}{4} x^{2}-\frac{\omega}{2}, \omega \in \mathbb{R}^{+} .
$$

With Dirichlet boundary conditions at infinity, it has the following spectrum $(z(x)=\sqrt{\omega / 2} x)$ :

$$
\left\{\begin{array}{c}
E_{n}(\omega)=n \omega \\
\psi_{n}(x ; \omega)=\psi_{0}(x ; \omega) \widetilde{H}_{n}(z)
\end{array}, \quad n \geq 0,\right.
$$

with

$$
\psi_{0}(x ; \omega)=\exp \left(-z^{2} / 2\right)
$$

and

$$
\widetilde{H}_{n}(z)=\frac{1}{2^{n}} H_{n}(z)
$$

the $H_{n}(z)$ being the classical Hermite polynomials.

It is the most simple example of TSIP, with $a=\omega \in \mathbb{R}$ and $\varepsilon=0$ (the parameter translation is of zero amplitude $a_{1}=\omega$ ), that is,

$$
V^{(0)}(x ; \omega)=V(x ; \omega)+\omega .
$$

\section{Isotonic oscillator}

The isotonic oscillator (IO) potential (with zero ground level $\left.E_{0}=0\right)$ ) is defined on the positive half line $] 0,+\infty[$ by

$$
V(x ; \omega, \alpha)=\frac{\omega^{2}}{4} x^{2}+\frac{(\alpha+1 / 2)(\alpha-1 / 2)}{x^{2}}-\omega(\alpha+1), \quad \alpha>1 / 2 .
$$


If we add Dirichlet boundary conditions at 0 and infinity and if we suppose $\alpha>1 / 2$, it has the following spectrum $\left(z(x)=\omega x^{2} / 2\right)$ :

$$
\left\{\begin{array}{c}
E_{n}(\omega)=2 n \omega \\
\psi_{n}(x ; \omega, \alpha)=\psi_{0}(x ; \omega, \alpha) \widetilde{L}_{n}^{\alpha}(z)
\end{array}, \quad n \geq 0,\right.
$$

where

$$
\psi_{0}(x ; \omega, \alpha)=z^{(\alpha+1 / 2) / 2} e^{-z / 2}
$$

and

$$
\widetilde{L}_{n}^{\alpha}(z)=(-1)^{n} n ! L_{n}^{\alpha}(z),
$$

the $L_{n}^{\alpha}(z)$ being the classical Laguerre polynomials.

It is a TSIP, with $a=(\omega, \alpha) \in \mathbb{R}^{2}$ and $\epsilon=(0,+1)$ :

$$
V^{(0)}(x ; \omega, \alpha)=V\left(x ; \omega, \alpha_{1}\right)+2 \omega .
$$

\section{Trigonometric Darboux-Pöschl-Teller potential}

The TDPT potential is defined on the interval $] 0, \pi / 2[$ by

$V(x ; \alpha, \beta)=\frac{(\alpha+1 / 2)(\alpha-1 / 2)}{\sin ^{2} x}+\frac{(\beta+1 / 2)(\beta-1 / 2)}{\cos ^{2} x}-(\alpha+\beta+1)^{2}, \alpha, \beta>1 / 2$.

With Dirichlet boundary conditions at 0 and $\pi / 2$, it has the following spectrum $(z(x)=\cos 2 x)$ :

$$
\left\{\begin{array}{c}
E_{n}(\alpha, \beta)=\left(\alpha_{n}+\beta_{n}+1\right)^{2}-(\alpha+\beta+1)^{2}=4 n(\alpha+\beta+1+n) \\
\psi_{n}(x ; \alpha, \beta)=\psi_{0}(x ; \alpha, \beta) \widetilde{P}_{n}^{(\alpha, \beta)}(z)
\end{array}, n \in \mathbb{N},\right.
$$

where

$$
\psi_{0}(x ; \alpha, \beta)=(\sin x)^{\alpha+1 / 2}(\cos x)^{\beta+1 / 2}
$$

and

$$
\widetilde{P}_{n}^{(\alpha, \beta)}(z)=\frac{2^{n} n !}{(\alpha+\beta+n+1)_{n}} P_{n}^{(\alpha, \beta)}(z) .
$$

The $P_{n}^{(\alpha, \beta)}$ are the usual Jacobi polynomials, $(x)_{n}=x(x+1) \ldots(x+n-1)$ is the Pochhammer symbol or rising factorial, ${ }^{17,18}$ and $\left(\alpha_{n}, \beta_{n}\right)=(\alpha+n, \beta+n)$.

It is a TSIP, with $a=(\alpha, \beta) \in \mathbb{R}^{2}$ and $\varepsilon=(+1,+1)$ :

$$
V^{(0)}(x ; \alpha, \beta)=V\left(x ; \alpha_{1}, \beta_{1}\right)+4(\alpha+\beta+2) \text {. }
$$

\section{B. Schur functions and Jacobi-Trudi formula}

In the symmetric functions theory, the Schur functions or Schur polynomials play a central role. ${ }^{19}$ These symmetric polynomials $S_{\lambda}(X)$, where $X=\left(x_{1}, \ldots, x_{m}\right)$ and where $\lambda=\left(\lambda_{1}, \ldots, \lambda_{m}\right)$ is any partition of length $m$, are defined as the ratio of two alternants ${ }^{24}$ whose elements are monomials in each of the variables $x_{i}$,

$$
S_{\lambda}(X)=\frac{\Delta_{\lambda}(X)}{\Delta_{0}(X)}
$$

with

$$
\Delta_{\lambda}(X)=\operatorname{det}\left(x_{i}^{\lambda_{j}+m-j}\right)_{1 \leq i<j \leq m}=\left|\begin{array}{ccc}
x_{1}^{\lambda_{1}+m-1} & \ldots & x_{m}^{\lambda_{1}+m-1} \\
x_{1}^{\lambda_{2}+m-2} & \ldots & x_{m}^{\lambda_{2}+m-2} \\
\ldots & & \ldots \\
x_{1}^{\lambda_{m}} & \ldots & x_{m}^{\lambda_{m}}
\end{array}\right|,
$$


$\Delta_{0}(X)$ being the Vandermondian

$$
\Delta_{0}(X)=\left|\begin{array}{ccc}
x_{1}^{m-1} & \ldots & x_{m}^{m-1} \\
\ldots & \ldots & \ldots \\
x_{1} & & x_{m} \\
1 & \ldots & 1
\end{array}\right|==_{1 \leq i<j \leq m}\left(x_{i}-x_{j}\right)
$$

We then have $\mathrm{e}^{20}$

$$
S_{\lambda}(X)=\sum_{T_{\lambda}} x_{1}^{t_{1}} \ldots x_{m}^{t_{m}},
$$

where the sum is over all the semi-standard Young tableaux $T_{\lambda}$ of weights $\left(t_{1}, \ldots, t_{m}\right)$ associated to $\lambda$.

The Schur polynomials of degree $d$ in the variables $\left(x_{1}, \ldots, x_{m}\right)$ form a basis of the space of the homogeneous symmetric polynomials of degree $d$ in the same variables. The $S_{\lambda}(X)$ can be obtained in terms of the complete symmetric polynomials $h_{k}(X)$ via the Jacobi-Trudy determinantal formula

$$
S_{\lambda}(X)=\operatorname{det}\left(h_{\lambda_{i}+j-i}\right)_{1 \leq i, j \leq m}=\left|\begin{array}{cccc}
h_{\lambda_{1}}(X) & h_{\lambda_{2}-1}(X) & \ldots & h_{\lambda_{m}-m+1}(X) \\
h_{\lambda_{1}+1}(X) & h_{\lambda_{2}}(X) & \ldots & h_{\lambda_{m}-m+2}(X) \\
\ldots & \ldots & & \ldots \\
h_{\lambda_{1}+m-1}(X) & h_{\lambda_{2}+m-2}(X) & \ldots & h_{\lambda_{m}}(X)
\end{array}\right|,
$$

where

$$
h_{k}(X)=\sum_{1 \leq i_{1} \leq \ldots \leq i_{k} \leq m} x_{i_{1}} \ldots x_{i_{k}}=\sum_{\substack{l_{i}=0 \\ l_{1}+\ldots+l_{m}=k}}^{m} x_{1}^{l_{1}} \ldots x_{m}^{l_{m}}
$$

In the approach developed by Noumi ${ }^{21}$ for the construction of the rational solutions of the Painlevé equations, the author proposed a determinantal formula for the so-called $\phi$-factors of these equations. They are special polynomials (as the Yablonski-Vorobev, Okamoto or generalized Hermite polynomials ${ }^{22,23}$ ) from which the rational solutions of Painlevé equations II and IV can be built. These authors proved that for each of these polynomials there is a partition $\lambda$ such that the polynomial can be written as

$$
\phi_{\lambda} \propto\left|\begin{array}{cccc}
g_{\lambda_{1}}^{(0)} & g_{\lambda_{2}-1}^{(0)} & \cdots & g_{\lambda_{m}-m+1}^{(0)} \\
g_{\lambda_{1}+1}^{(-1)} & g_{\lambda_{2}}^{(-1)} & \cdots & g_{\lambda_{m}-m+2}^{(-1)} \\
\ldots & \ldots & & \ldots \\
g_{\lambda_{1}+m-1}^{(-m+1)} & g_{\lambda_{2}+m-2}^{(-m+1)} & \cdots & g_{\lambda_{m}}^{(-m+1)}
\end{array}\right|
$$

the $g_{j}^{(i)}$ being polynomials associated to the particular partitions which correspond to one-row Young diagrams.

Although the entries in the determinant above depend on an additional index associated with the row, the kinship between the structures of the two expressions Eqs. (45) and (47) is clear and Noumi and Yamada call Eq. (47) the Jacobi-Trudi formula for the $\phi$-factors. As in the case of the original Jacobi-Trudi formula Eq. (45), it allows to express functions associated with general Young diagrams in terms of those associated with one-row diagrams via a determinantal formula with a specific structure. In Noumi-Yamada's approach, if the $g_{j}^{(i)}$ are initially seen as polynomials in many variables, these last are then specialized to obtain polynomials in only one-variable, the specialization choice depending on the considered Painlevé equation. 


\section{Jacobi-Trudi type formula for exceptional orthogonal polynomials}

Using the Crum formulas, Eqs. (13) and (14), the form of the extensions obtained from a PTSIP via chains of SDDBT as well as their eigenfunctions are determined by Wronskians of the type

$$
W^{\left(N_{m}\right)}(x ; a)=W\left(\psi_{n_{1}}(x ; a), \ldots, \psi_{n_{m}}(x ; a) \mid x\right),
$$

where the $\psi_{n}$ are given by Eq. (21). $W^{\left(N_{m}\right)}$ is characterized by the $m$-tuple of spectral indices $N_{m}$ $=\left(n_{1}, \ldots, n_{m}\right)\left(n_{m}>\ldots>n_{1} \geq 0\right)$ or equivalently by the associated partition $\lambda=\left(\lambda_{1}, \ldots, \lambda_{m}\right)$ (see Eq. (15)). Considering the three confining PTSIP mentioned above and using the standard properties of Wronskians, ${ }^{24}$ this can be rewritten as

$$
W^{\left(N_{m}\right)}(x ; a)=\left(\psi_{0}(x ; a)\right)^{m} W\left(P_{n_{1}}^{a}(z), \ldots, P_{n_{m}}^{a}(z) \mid x\right)=\left(\psi_{0}(x ; a)\right)^{m}\left(\frac{d z}{d x}\right)^{m(m-1) / 2} \mathcal{W}_{\lambda}(z),
$$

where (see Eq. (15))

$$
\mathcal{W}_{\lambda}(z)=W\left(P_{n_{1}}^{a}(z), \ldots, P_{n_{m}}^{a}(z) \mid z\right)=W\left(P_{\lambda_{m}}^{a}(z), \ldots, P_{\lambda_{1}+m-1}^{a}(z) \mid z\right)
$$

is a polynomial in $z$ that we call in an abusive manner an EOP enlarging the denomination associated to regular extensions. ${ }^{1}$ Since, as we have seen above, the regularity is only related to a particular structure of the partition $\lambda$, we do not refer to it in the following.

For the monic classical orthogonal polynomials, we have ${ }^{17,18}$

$$
\frac{d}{d z} P_{n}^{a}(z)=n P_{n-1}^{a_{1}}(z)
$$

which gives

$$
\mathcal{W}_{\lambda}(z)=\left|\begin{array}{cccc}
A_{\lambda_{m}}^{0} P_{\lambda_{m}}^{a}(z) & A_{\lambda_{m-1}+1}^{0} P_{\lambda_{m-1}+1}^{a}(z) & \ldots & A_{\lambda_{1}+m-1}^{0} P_{\lambda_{1}+m-1}^{a}(z) \\
A_{\lambda_{m}}^{1} P_{\lambda_{m}-1}^{a_{1}}(z) & A_{\lambda_{m-1}+1}^{1} P_{\lambda_{m-1}}^{a_{1}}(z) & \ldots & A_{\lambda_{1}+m-1}^{1} P_{\lambda_{1}+m-2}^{a_{1}}(z) \\
\ldots & \ldots & & \ldots \\
A_{\lambda_{m}}^{m-1} P_{\lambda_{m}-m+1}^{a_{m-1}}(z) & A_{\lambda_{m-1}+1}^{m-1} P_{\lambda_{m-1}-m+2}^{a_{m-1}}(z) & \ldots & A_{\lambda_{1}+m-1}^{m-1} P_{\lambda_{1}}^{a_{m-1}}(z)
\end{array}\right|,
$$

where

$$
A_{n}^{k}=n^{\underline{k}}=n(n-1) \ldots(n-k+1)=k ! C_{n}^{k}
$$

is the falling factorial i.e., "the number of arrangements of $k$ objects among $n . "$

Defining

$$
g_{k}^{(j)}(z)=A_{k+m-1-j}^{m-1-j} P_{k}^{a_{m-1}+j}(z)=\frac{(k-1+m-j) !}{k !} P_{k}^{a_{m-1}+j}(z)
$$

and reversing the order of the columns and of the lines, we arrive to

$$
\mathcal{W}_{\lambda}(z)=\left|\begin{array}{cccc}
g_{\lambda_{1}}^{(0)}(z) & g_{\lambda_{2}-1}^{(0)}(z) & \ldots & g_{\lambda_{m}-m+1}^{(0)}(z) \\
g_{\lambda_{1}+1}^{(1)}(z) & g_{\lambda_{2}}^{(1)}(z) & \ldots & g_{\lambda_{m}-m+2}^{(1)}(z) \\
\ldots & \ldots & & \ldots \\
g_{\lambda_{1}+m-1}^{(m-1)}(z) & g_{\lambda_{2}+m-2}^{(m-1)}(z) & \ldots & g_{\lambda_{m}}^{(m-1)}(z)
\end{array}\right|
$$

Consequently, the EOP $\mathcal{W}_{\lambda}(z)$ is amenable to a Jacobi-Trudi type formula (see Eq. (47)), analogous to the one satisfied by the $\phi$-factors in Noumi-Yamada approach of the rational solutions of the Painlevé equations. It has to be noticed that the generalized Hermite and Okamoto polynomials ${ }^{22,23}$ appear as particular exceptional Hermite polynomials in the enlarged sense given above. 


\section{WRONSKIANS AND CONFLUENT ALTERNANTS}

Consider the following alternant

$$
\Delta(\Phi \mid X)=\left|\begin{array}{ccc}
\phi_{1}\left(x_{1}\right) & \ldots & \phi_{1}\left(x_{m}\right) \\
\ldots & & \ldots \\
\phi_{m}\left(x_{1}\right) & \ldots & \phi_{m}\left(x_{m}\right)
\end{array}\right|,
$$

where the $\phi_{i}$ are supposed polynomials of degree $n_{i}$ and where we have noted $\Phi=\left(\phi_{1}, \ldots, \phi_{m}\right)$ and $X=\left(x_{1}, \ldots, x_{m}\right)$. For the case $\phi_{k}(x)=x^{k-1}$ the preceding determinant reduces to the Vandermondian $\Delta(X) . \Delta(\Phi \mid X)$ being a polynomial antisymmetric in the exchange of two variables $x_{i}$ and $x_{j}$, it is divisible by $\Delta_{0}(X)$ and the ratio $\frac{\Delta(\Phi \mid X)}{\Delta_{0}(X)}$ is a symmetric polynomial $S(\Phi \mid X)$.

In the case where the $\phi_{k}$ are monic polynomials of respective degrees $k$, then by linear combinations of the columns, we obtain also

$$
\Delta(\Phi \mid X)=\Delta_{0}(X) .
$$

We are interested in the confluent limit $x_{i} \rightarrow x, \forall i \in\{1, \ldots, m\}$. Defining the new set of variable $\varepsilon_{k}$ via $x_{k}=x+\varepsilon_{k}$, we have

$$
\phi_{i}\left(x+\varepsilon_{j}\right)=\sum_{k=0}^{n_{i}} a_{k, i}(x) \varepsilon_{j}^{k}=p_{i}\left(\varepsilon_{j}\right),
$$

with

$$
a_{k, i}(x)=\phi_{i}^{(k)}(x) / k !
$$

$\left(a_{k, i}(x)=0\right.$ if $\left.k>n_{i}\right)$ and

$$
\Delta(\Phi \mid X)=\left|\begin{array}{ccc}
p_{1}\left(\varepsilon_{1}\right) & \ldots & p_{1}\left(\varepsilon_{m}\right) \\
\ldots & & \ldots \\
p_{m}\left(\varepsilon_{1}\right) & \ldots & p_{m}\left(\varepsilon_{m}\right)
\end{array}\right|=\Delta(P \mid \varepsilon),
$$

where $P=\left(p_{1}, \ldots, p_{m}\right)$ and $\varepsilon=\left(\varepsilon_{1}, \ldots, \varepsilon_{m}\right)$. Moreover,

$$
\Delta(X)=_{1 \leq i<j \leq m}\left(\varepsilon_{i}-\varepsilon_{j}\right)=\Delta(\varepsilon),
$$

which implies

$$
S(\Phi \mid X)=S(P \mid \varepsilon) .
$$

The ratio $S(P \mid \varepsilon)=\Delta(P \mid \varepsilon) / \Delta(\varepsilon)$ is also a symmetric polynomial and is consequently a continuous function of $\varepsilon$ on $\mathbb{R}^{m}$. It results in particular that to calculate the value $S(P \mid 0)$, we can successively apply the limits $\varepsilon_{1} \rightarrow 0, \varepsilon_{2} \rightarrow 0, \ldots, \varepsilon_{m} \rightarrow 0$ in this order. We then have the following result:

Theorem 1.

In the confluent limit $x_{i} \rightarrow x, \forall i \in\{1, \ldots, m\}$,

$$
S(\Phi \mid X)=\frac{\Delta(\Phi \mid X)}{\Delta(X)} \underset{\left\{x_{i} \rightarrow x\right\}}{\rightarrow} \frac{W\left(\phi_{1}, \ldots, \phi_{m} \mid x\right)}{\underset{j=1}{m-1} j !} .
$$

Proof.

From Eqs. (62) and (58), we have

$$
S(\Phi \mid X)=S(P \mid \varepsilon)=\frac{1}{\Delta(\varepsilon)}\left|\begin{array}{ccc}
n_{1} a_{k, 1}(x) \varepsilon_{1}^{k} & \ldots & n_{k=0}^{n_{1}} a_{k, 1}(x) \varepsilon_{m}^{k} \\
\ldots & & \ldots \\
n_{m=0} a_{k, m}(x) \varepsilon_{1}^{k} & \ldots & n_{m=0} a_{k, m}(x) \varepsilon_{m}^{k}
\end{array}\right|,
$$


or by subtracting the first column to the following ones:

$$
\begin{aligned}
S(\Phi \mid X)= & \frac{1}{\Delta\left(\varepsilon_{1}, \ldots, \varepsilon_{m}\right)} \\
& \times\left|\begin{array}{cccc}
a_{0,1}(x)+O\left(\varepsilon_{1}\right) & { }_{k=1}^{n_{1}} a_{k, 1}(x)\left(\varepsilon_{2}^{k}-\varepsilon_{1}^{k}\right) & \ldots & { }_{k=1}^{n_{1}} a_{k, 1}(x)\left(\varepsilon_{m}^{k}-\varepsilon_{1}^{k}\right) \\
\ldots & \ldots & \ldots \\
a_{0, m}(x)+O\left(\varepsilon_{1}\right) & \substack{k=0 \\
k}_{k, m} a_{k, m}(x)\left(\varepsilon_{2}^{k}-\varepsilon_{1}^{k}\right) & \ldots & { }_{k=0}^{n_{m}} a_{k, m}(x)\left(\varepsilon_{m}^{k}-\varepsilon_{1}^{k}\right)
\end{array}\right|,
\end{aligned}
$$

that is,

$$
\begin{aligned}
S(\Phi \mid X)= & \frac{1}{\Delta\left(\varepsilon_{2}, \ldots, \varepsilon_{m}\right)} \\
& \times\left|\begin{array}{cccc}
a_{0,1}(x)+O\left(\varepsilon_{1}\right) & { }_{k=1}^{n_{1}} a_{k, 1}(x) \varepsilon_{2}^{k-1}\left(1+O\left(\varepsilon_{1}\right)\right) & \ldots & { }_{k=1}^{n_{1}} a_{k, 1}(x) \varepsilon_{m}^{k-1}\left(1+O\left(\varepsilon_{1}\right)\right) \\
\ldots & \ldots & \ldots \\
a_{0, m}(x)+O\left(\varepsilon_{1}\right) & { }_{k=1}^{n_{m}} a_{k, m}(x) \varepsilon_{2}^{k-1}\left(1+O\left(\varepsilon_{1}\right)\right) & \ldots & { }_{k=1}^{n_{m}} a_{k, m}(x) \varepsilon_{m}^{k-1}\left(1+O\left(\varepsilon_{1}\right)\right)
\end{array}\right| .
\end{aligned}
$$

If we take the limit $\varepsilon_{1} \rightarrow 0$, i.e., $x_{1} \rightarrow x$, we obtain

$$
\lim _{x_{1} \rightarrow x} S(\Phi \mid X)=\frac{1}{\Delta\left(\varepsilon_{2}, \ldots, \varepsilon_{m}\right)}\left|\begin{array}{cccc}
a_{0,1}(x) & \begin{array}{c}
n_{1} \\
k=1
\end{array} a_{k, 1}(x) \varepsilon_{2}^{k-1} & \ldots & n_{k=1}^{n_{1}} a_{k, 1}(x) \varepsilon_{m}^{k-1} \\
\ldots & \ldots & & \ldots \\
a_{0, m}(x) & { }_{k=1}^{n_{m}} a_{k, m}(x) \varepsilon_{2}^{k-1} & \ldots & { }_{k=1}^{n_{m}} a_{k, m}(x) \varepsilon_{m}^{k-1}
\end{array}\right| .
$$

Subtracting the second columns to the following ones gives

$$
\begin{aligned}
& S\left(\Phi \mid x, x_{2}, \ldots, x_{m}\right) \\
& =\frac{1}{\Delta\left(\varepsilon_{2}, \ldots, \varepsilon_{m}\right)}\left|\begin{array}{cccc}
a_{0,1}(x) & a_{1,1}(x)+O\left(\varepsilon_{2}\right) & \ldots & { }_{k=2}^{n_{1}} a_{k, 1}(x)\left(\varepsilon_{m}^{k-1}-\varepsilon_{2}^{k-1}\right) \\
\ldots & \ldots & & \ldots \\
a_{0, m}(x) & a_{1, m}(x)+O\left(\varepsilon_{2}\right) & \ldots & { }_{k=2}^{n_{m}} a_{k, m}(x)\left(\varepsilon_{m}^{k-1}-\varepsilon_{2}^{k-1}\right)
\end{array}\right| \\
& =\frac{1}{\Delta\left(\varepsilon_{3}, \ldots, \varepsilon_{m}\right)}\left|\begin{array}{cccc}
a_{0,1}(x) & a_{1,1}(x)+O\left(\varepsilon_{2}\right) & \ldots & { }_{k=2}^{n_{1}} a_{k, 1}(x) \varepsilon_{m}^{k-2}\left(1+O\left(\varepsilon_{2}\right)\right) \\
\ldots & \ldots & & \ldots \\
a_{0, m}(x) & a_{1, m}(x)+O\left(\varepsilon_{2}\right) & \ldots & { }_{k=2}^{n_{m}} a_{k, m}(x) \varepsilon_{m}^{k-2}\left(1+O\left(\varepsilon_{2}\right)\right)
\end{array}\right|
\end{aligned}
$$

and

$$
\begin{aligned}
S\left(\Phi \mid x, x, x_{3}, \ldots, x_{m}\right)= & \lim _{x_{2} \rightarrow x} S\left(\Phi \mid x, x_{2}, \ldots, x_{m}\right)=\frac{1}{\Delta\left(\varepsilon_{3}, \ldots, \varepsilon_{m}\right)} \\
& \times\left|\begin{array}{cccc}
a_{0,1}(x) & a_{1,1}(x) & \ldots & { }_{k=2}^{n_{1}} a_{k, 1}(x) \varepsilon_{m}^{k-2} \\
\ldots & \ldots & & \ldots \\
a_{0, m}(x) & a_{1, m}(x) & \ldots & { }_{k=2}^{n_{m}} a_{k, m}(x) \varepsilon_{m}^{k-2}
\end{array}\right| .
\end{aligned}
$$

Repeating $m$ times the above procedure, we obtain in the limit $x_{i} \rightarrow x$ (or $\varepsilon_{i} \rightarrow 0$ ), $\forall i \in\{1, \ldots$, $m\}$,

$$
S(\Phi \mid x, \ldots, x)=\left|\begin{array}{cccc}
a_{0,1}(x) & a_{1,1}(x) & \ldots & a_{m-1,1}(x) \\
\ldots & \ldots & & \ldots \\
a_{0, m}(x) & a_{1, m}(x) & \ldots & a_{m-1, m}(x)
\end{array}\right|=\frac{W\left(\phi_{1}, \ldots, \phi_{m} \mid x\right)}{\underset{j=1}{m-1} j !},
$$

where we have used Eq. (59). 


\section{CONFLUENT LIMITS OF THE GENERALIZED SCHUR POLYNOMIALS AND OF THE GENERALIZED JACOBI-TRUDI FORMULA}

\section{A. Exceptional orthogonal polynomials and generalized Schur polynomials}

In Ref. 5, Sergeev and Veselov have generalized the Schur polynomials in the following way: starting from a family of monic polynomials $\left(P_{n}^{a}\right)$ satisfying a three term recurrence relation (as for instance the classical orthogonal polynomials given in Eqs. (29), (34), and (39)), they replace in Eq. (45) the monomials $x_{i}^{\lambda_{j}+m-j}$ by the polynomials $P_{\lambda_{j}+m-j}^{\alpha}\left(z_{i}\right)$,

$$
S_{\lambda}(Z)=\operatorname{det}\left(P_{\lambda_{j}+m-j}^{\alpha}\left(z_{i}\right)\right)_{1 \leq i<j \leq m}=\frac{\Delta_{\lambda}(P \mid Z)}{\Delta_{0}(P \mid Z)},
$$

where 5,6

$$
\Delta_{\lambda}(P \mid Z)=\left|\begin{array}{ccc}
P_{\lambda_{1}+m-1}^{\alpha}\left(z_{1}\right) & \ldots & P_{\lambda_{1}+m-1}^{\alpha}\left(z_{m}\right) \\
\ldots & & \ldots \\
P_{\lambda_{m}}^{\alpha}\left(z_{1}\right) & \ldots & P_{\lambda_{m}}^{\alpha}\left(z_{m}\right)
\end{array}\right| .
$$

Note that (see Eq. (57))

$$
\Delta_{0}(P \mid Z)=\Delta_{0}(Z) .
$$

From Theorem 1 and Eq. (63), we then deduce immediately (see Eq. (50))

$$
S_{\lambda}(z)=\lim _{\left\{z_{i} \rightarrow z\right\}} S_{\lambda}(Z)=\frac{1}{\substack{m-1 \\ j=1}} \mathcal{W}_{\lambda}(z),
$$

and the EOP considered above appear to be the confluent limits of generalized Schur polynomials.

\section{B. Confluent limit of the generalized Jacobi-Trudi formula}

Sergeev and Veselov ${ }^{5,6}$ have shown that the generalized Schur polynomials satisfy a generalized Jacobi-Trudi formula of the form

$$
S_{\lambda}(Z)=\left|\begin{array}{cccc}
S_{\lambda_{1}}^{(0, m)}(Z) & S_{\lambda_{1}}^{(1, m)}(Z) & \ldots & S_{\lambda_{1}}^{(m-1, m)}(Z) \\
S_{\lambda_{2}-1}^{(0, m)}(Z) & S_{\lambda_{2}-1}^{(1, m)}(Z) & \ldots & S_{\lambda_{2}-1}^{(m-1, m)}(Z) \\
\ldots & \ldots & & \ldots \\
S_{\lambda_{m}-m+1}^{(0, m)}(Z) & S_{\lambda_{m}-m+1}^{(1, m)}(Z) & \ldots & S_{\lambda_{m}-m+1}^{(m-1, m)}(Z)
\end{array}\right|,
$$

where the multivariable polynomials $S_{k}^{(i, m)}(Z)$ verify the recursion relations

$$
\left\{\begin{array}{c}
S_{k}^{(i+1, m)}(Z)=S_{k+1}^{(i, m)}(Z)+p_{k+m-1, a} S_{k}^{(i, m)}(Z)+q_{k+m-1, a} S_{k-1}^{(i, m)}(Z) \\
S_{k}^{(i+1, m)}(Z)=z_{1} S_{k}^{(i, m)}(Z)+S_{k+1}^{(i, m-1)}(Z)
\end{array},\right.
$$

the coefficients $p_{k}$ and $q_{k}$ being given, respectively, by Eqs. (23), (24), and (25).

$S_{k \geq 0}^{(0, m)}(Z)$ is the generalized Schur polynomial associated to a column Young diagram of height $k$, that is,

$$
S_{k}^{(0, m)}(Z)=S_{(k, 0, \ldots, 0)}(Z)=\frac{1}{\Delta(Z)}\left|\begin{array}{ccc}
P_{k+m-1}^{a}\left(z_{1}\right) & \ldots & P_{k+m-1}^{a}\left(z_{m}\right) \\
P_{m-2}^{a}\left(z_{1}\right) & \ldots & P_{m-2}^{a}\left(z_{m}\right) \\
\ldots & & \ldots \\
P_{0}^{a}\left(z_{1}\right) & \ldots & P_{0}^{a}\left(z_{m}\right)
\end{array}\right|
$$

which is extended to negative $k$ by setting $S_{k<0}^{(0, m)}(Z)=0$. 
The confluent limit of the generalized Jacobi-Trudy formula Eq. (75),

$$
S_{\lambda}(z)=\left|\begin{array}{cccc}
S_{\lambda_{1}}^{(0, m)}(z) & S_{\lambda_{1}}^{(1, m)}(z) & \ldots & S_{\lambda_{1}}^{(m-1, m)}(z) \\
S_{\lambda_{2}-1}^{(0, m)}(z) & S_{\lambda_{2}-1}^{(1, m)}(z) & \ldots & S_{\lambda_{2}-1}^{(m-1, m)}(z) \\
\ldots & \ldots & & \ldots \\
S_{\lambda_{m}-m+1}^{(0, m)}(z) & S_{\lambda_{m}-m+1}^{(1, m)}(z) & \ldots & S_{\lambda_{m}-m+1}^{(m-1, m)}(z)
\end{array}\right|
$$

is a priori different in nature from the Jacobi-Trudi type formula given in Eq. (55). This last does not appear as the confluent limit of Sergeev-Veselov's generalized Jacobi-Trudi formula but simply as a rewriting of Eq. (74) on the basis of the explicit derivation formula Eq. (51). More precisely, in the confluent limit, we deduce immediately from Theorem 1,

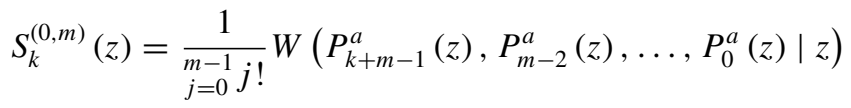

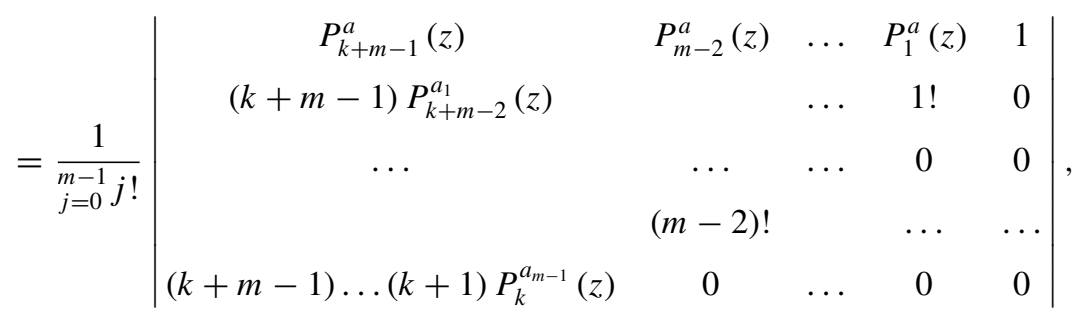

that is, using Eq. (51):

$$
S_{k}^{(0, l)}(z)=\left(\begin{array}{c}
k+l-1 \\
k
\end{array}\right) P_{k}^{a_{l-1}}(z)=\frac{1}{(l-1) !} \frac{d^{l-1}}{d z^{l-1}}\left(P_{k+l-1}^{a}(z)\right)
$$

The $g_{k}^{(j)}(z)$ functions appearing in Eq. (55) identify then to the $S_{k}^{(0, l)}(z)$ rather than to the $S_{k}^{(j, l)}(z)$.

The connection between the two Jacobi-Trudy type formulas becomes clearer if we return to the proof of Eq. (78) in the specific confluent case. If we note

$$
\mathbf{S}_{\lambda}^{(i, m)}(z)=\left(\begin{array}{c}
S_{\lambda_{1}}^{(i, m)}(z) \\
\ldots \\
S_{\lambda_{m}-m+1}^{(i, m)}(z)
\end{array}\right)
$$

we have

$$
S_{\lambda}(z)=\operatorname{det}\left[\mathbf{S}_{\lambda}^{(0, m)}(z), \mathbf{S}_{\lambda}^{(1, m)}(z), \ldots, \mathbf{S}_{\lambda}^{(m-1, m)}(z)\right]
$$

Equation (76) implies then

$$
\mathbf{S}_{\lambda}^{(i+1, m)}(z)={ }_{z} \mathbf{S}_{\lambda}^{(i, m)}(z)+\mathbf{S}_{\lambda+1}^{(i, m-1)}(z),
$$


where $\lambda+1=\left(\lambda_{1}+1, \ldots, \lambda_{m}+1\right)$. By successively using Eq. (83) and combining the columns from the last one to the second one, we obtain

$$
\begin{aligned}
S_{\lambda}(z) & =\operatorname{det}\left[\mathbf{S}_{\lambda}^{(0, m)}(z), \ldots, \mathbf{S}_{\lambda}^{(m-3, m)}(z), \mathbf{S}_{\lambda}^{(m-2, m)}(z), z \mathbf{S}_{\lambda}^{(m-2, m)}(z)+\mathbf{S}_{\lambda+1}^{(m-2, m-1)}(z)\right] \\
& =\operatorname{det}\left[\mathbf{S}_{\lambda}^{(0, m)}(z), \ldots, \mathbf{S}_{\lambda}^{(m-3, m)}(z), \mathbf{S}_{\lambda}^{(m-2, m)}(z), \mathbf{S}_{\lambda+1}^{(m-2, m-1)}(z)\right] \\
& =\operatorname{det}\left[\mathbf{S}_{\lambda}^{(0, m)}(z), \ldots, \mathbf{S}_{\lambda}^{(m-3, m)}(z), z \mathbf{S}_{\lambda}^{(m-3, m)}(z)+\mathbf{S}_{\lambda+1}^{(m-3, m-1)}(z), \mathbf{S}_{\lambda+1}^{(m-2, m-1)}(z)\right] \\
& =\operatorname{det}\left[\mathbf{S}_{\lambda}^{(0, m)}(z), \ldots, \mathbf{S}_{\lambda}^{(m-3, m)}(z), \mathbf{S}_{\lambda+1}^{(m-3, m-1)}(z), \mathbf{S}_{\lambda+1}^{(m-2, m-1)}(z)\right] \\
& =\ldots \\
& =\operatorname{det}\left[\mathbf{S}_{\lambda}^{(0, m)}(z), \mathbf{S}_{\lambda+1}^{(0, m-1)}(z), \mathbf{S}_{\lambda+1}^{(1, m-1)}(z), \ldots, \mathbf{S}_{\lambda+1}^{(m-2, m-1)}(z)\right] .
\end{aligned}
$$

Repeating the same procedure on the columns from the last one to the third one gives

$$
S_{\lambda}(z)=\operatorname{det}\left[\mathbf{S}_{\lambda}^{(0, m)}(z), \mathbf{S}_{\lambda+1}^{(0, m-1)}(z), \mathbf{S}_{\lambda+2}^{(0, m-2)}(z), \mathbf{S}_{\lambda+2}^{(1, m-2)}(z), \ldots, \mathbf{S}_{\lambda+2}^{(m-3, m-2)}(z)\right] .
$$

The recursion is immediate and we obtain

$$
\begin{aligned}
S_{\lambda}(z) & =\operatorname{det}\left[\mathbf{S}_{\lambda}^{(0, m)}(z), \mathbf{S}_{\lambda+1}^{(0, m-1)}(z), \mathbf{S}_{\lambda+2}^{(0, m-2)}(z), \ldots, \mathbf{S}_{\lambda+m-1}^{(0,1)}(z)\right] \\
& =\left|\begin{array}{cccc}
S_{\lambda_{1}}^{(0, m)}(z) & S_{\lambda_{1}+1}^{(0, m-1)}(z) & \ldots & S_{\lambda_{1}+m-1}^{(0,1)}(z) \\
S_{\lambda_{2}-1}^{(0, m)}(z) & S_{\lambda_{2}}^{(0, m-1)}(z) & \ldots & S_{\lambda_{2}+m-2}^{(0,1)}(z) \\
\ldots & \ldots & & \ldots \\
S_{\lambda_{m}-m+1}^{(0, m)}(z) & S_{\lambda_{m}-m+2}^{(0, m-1)}(z) & \ldots & S_{\lambda_{m}}^{(0,1)}(z)
\end{array}\right|,
\end{aligned}
$$

that is,

$$
S_{\lambda}(z)=\left|\begin{array}{cccc}
\frac{1}{(m-1) !} \frac{d^{m-1}}{z^{m-1}}\left(P_{\lambda_{1}+m-1}^{a}(z)\right) & \frac{1}{(m-2) !} \frac{d^{m-2}}{d z^{m-2}}\left(P_{\lambda_{1}+m-1}^{a}(z)\right) & \ldots & P_{\lambda_{1}+m-1}^{a}(z) \\
\frac{1}{(m-1) !} \frac{d^{m-1}}{d z^{m-1}}\left(P_{\lambda_{2}+m-2}^{a}(z)\right) & \frac{1}{(m-2) !} \frac{d^{m-2}}{d z^{m-2}}\left(P_{\lambda_{2}+m-2}^{a}(z)\right) & \ldots & P_{\lambda_{2}+m-2}^{a}(z) \\
\ldots & \ldots & & \ldots \\
\frac{1}{(m-1) !} \frac{d^{m-1}}{d z^{m-1}}\left(P_{\lambda_{m}}^{a}(z)\right) & \frac{1}{(m-2) !} \frac{d^{m-2}}{d z^{m-2}}\left(P_{\lambda_{m}}^{a}(z)\right) & \ldots & P_{\lambda_{m}}^{a}(z)
\end{array}\right| .
$$

We finally recover Eq. (74) and consequently Eq. (55).

\section{ACKNOWLEDGMENTS}

I would like to thank R. Milson and D. Gomez-Ullate for fruitful exchanges and helpful suggestions. Thanks also to J. Harnad for stimulating discussions.

${ }^{1}$ D. Gomez-Ullate, N. Kamran, and R. Milson, “An extension of Bochner's problem: Exceptional invariant subspaces," J. Approx. Theory 162, 987-1006 (2010).

${ }^{2}$ D. Gomez-Ullate, N. Kamran, and R. Milson, "An extended class of orthogonal polynomials defined by a Sturm-Liouville problem," J. Math. Anal. Appl. 359, 352-357 (2009).

${ }^{3}$ D. Gomez-Ullate, Y. Grandati, and R. Milson, "Extended Krein-Adler theorem for the translationally shape invariant potentials," J. Math. Phys. 55, 043510 (2014).

${ }^{4}$ D. Gomez-Ullate, Y. Grandati, and R. Milson, "Rational extensions of the quantum harmonic oscillator and exceptional Hermite polynomials," J. Phys. A: Math. Theor. 47, 015203 (2014).

${ }^{5}$ A. N. Sergeev and A. P. Veselov, "Jacobi-Trudi formula for generalized Schur polynomials," preprint arXiv:0905.2557 (2009).

${ }^{6} \mathrm{~J}$. Harnad and E. Lee, "Symmetric polynomials generalized Jacobi-Trudi identities and $\tau$-functions," preprint arXiv: 1304.0020 (2013).

${ }^{7}$ M. M. Crum, “Associated Sturm-Liouville systems," Q. J. Math. 6, 121-127 (1955).

${ }^{8}$ V. B. Matveev and M. A. Salle, Darboux Transformations and Solitons (Springer-Verlag, Berlin, 1991). 
${ }^{9}$ G. Felder, A. D. Hemery, and A. P. Veselov, "Zeros of Wronskians of Hermite polynomials and Young diagrams," Physica D 241, 2131-2137 (2012).

${ }^{10}$ M. G. Krein, "On a continuous analogue of a Christoffel formula from the theory of orthogonal polynomials," Dokl. Akad. Nauk SSSR 113, 970 (1957).

${ }^{11}$ V. E. Adler, "A modification of Crum method," Theor. Math. Phys. 101, 1381-1386 (1994).

${ }^{12}$ F. Cooper, A. Khare, and U. Sukhatme, Supersymmetry in Quantum Mechanics (World Scientific, Singapore, 2001).

${ }^{13}$ R. Dutt, A. Khare, and U. P. Sukhatme, "Supersymmetry, shape invariance and exactly solvable potentials," Am. J. Phys. 56, 163-168 (1988).

${ }^{14}$ L. Gendenshtein, "Derivation of exact spectra of the Schrodinger equation by means of supersymmetry," JETP Lett. 38, 356-359 (1983).

${ }^{15}$ Y. Grandati and A. Bérard, "Rational solutions for the Riccati-Schrödinger equations associated to translationally shape invariant potentials," Ann. Phys. 325, 1235-1259 (2010).

${ }^{16}$ Y. Grandati, "Multistep DBT and regular rational extensions of the isotonic oscillator," Ann. Phys. 327, 2411 (2012).

${ }^{17}$ G. Szegö, Orthogonal Polynomials (American Mathematical Society, Providence, 1975).

${ }^{18}$ A. Erdélyi, W. Magnus, F. Oberhettinger, and F. G. Tricomi, Higher Transcendental Functions (McGraw-Hill, New York, 1953).

${ }^{19}$ I. G. Macdonald, Symmetric Functions and Hall Polynomials (Oxford University Press, Oxford, 1995).

${ }^{20}$ W. Fulton and J. Harris, Representation Theory (Springer, Berlin, 1991).

${ }^{21}$ M. Noumi, Painlevé Equations through Symmetry (AMS, Providence, 2004).

${ }^{22}$ P. A. Clarkson, "Vortices and polynomials," Studies Appl. Math. 123, 37-62 (2009).

${ }^{23}$ G. V. Filipuk and P. A. Clarkson, "The symmetric fourth Painlevé hierarchy and associated special polynomials," Studies Appl. Math. 121, 157-188 (2008).

${ }^{24}$ T. Muir (revised and enlarged by W. H. Metzler), A Treatise on the Theory of Determinants (Dover, New York, 1960). 